\title{
Local Orbit Types of $S$-representations of Symmetric R-spaces
}

\author{
Kei KONDO \\ Saga University \\ (Communicated by R. Miyaoka) \\ Dedicated to Professor Katuhiro SHIOHAMA on his sixtieth birthday
}

E. Heintze and C. Olmos [HeiOlm] have investigated the local orbit types of $s$-representation of semisimple symmetric spaces in terms of restricted root systems. Their results have been generalized by H. Tamaru [Tama2]. But, as far as the author knows, there are no complete lists of all orbit types of $s$-representations of Riemannian symmetric spaces. The main purpose of this paper is to obtain a complete list of all local orbit types of $s$-representations of the following "symmetric R-spaces"; (i) the classical types of the rank 2: $T \cdot A I_{2}, T \cdot A I_{2}$, $A I I I_{2}, B D I_{2}, C I_{2}, C I_{2}, C I_{2}=G r_{2}\left(\mathbf{H}^{4}\right), D I I I_{2}$, (ii) the classical types of the rank 3: $T \cdot A I_{3}, T \cdot A I_{3}, A I_{3}, B D I_{3}, C_{3}, C I_{3}, D I_{3}$, (iii) the exceptional types: EIII, EIV, $E V I I, F I I=P^{2}(\mathbf{O}) ; G=G_{2} / S O(4)$ (as a normal space), (iv) the classical groups of the rank 2: $S O(4), S O(5), U(3), S p(2)$, (v) the classical groups of the rank 3: $S O(6), S O(7)$, $U(4), S p(3)$, (vi) the real quadrics: $S^{p} \cdot S^{q}(p \leq q)$, which is our main results (see Section 3). For a compact semisimple symmetric space, we get the result stated in Section 2 as follows;

THEOREM 0.1 (Criterion theorem 2.6 in Section 2). Any two orbits of a compact semisimple symmetric space are locally diffeomorphic if and only if their closed subsystems in the restricted root system are conjugate.

COROLLARY 0.2 (Corollary 2.8 in Section 2). The number of the local orbit types of $s$-representations of a compact semisimple symmetric space is less than or equal to $2^{r}$, where $r$ is the rank of the symmetric space.

Let $M=G / K$ be a compact semisimple symmetric space, where $G$ is the identity component of the isometry group. Let $H, H^{\prime}$ be two points in the tangent space $T_{o} M$ to $M$ at the origin $o \in M$, and let $K_{H}$ and $K_{H^{\prime}}$ be the isotropy subgroups of $K$ (identified with the linear isotropy group) at $H$ and $H^{\prime}$, respectively. We denote by $\mathfrak{k}_{H}$ and $\mathfrak{k}_{H^{\prime}}$ the Lie algebras of $K_{H}$ and $K_{H^{\prime}}$, respectively. We say that two orbits $K(H)=K / K_{H}$ and $K\left(H^{\prime}\right)=K / K_{H^{\prime}}$ are of the same orbit type if $K_{H}$ is conjugate to $K_{H^{\prime}}$ in $K$ under the automorphism group of $K$. Thus, we say that two orbits $K(H)=K / K_{H}$ and $K\left(H^{\prime}\right)=K / K_{H^{\prime}}$ are of the same local orbit type if $\mathfrak{k}_{H}$ is conjugate to $\mathfrak{k}_{H^{\prime}}$ in $\mathfrak{k}$ under the automorphism group of $\mathfrak{k}$. We say that

Received November 22, 2001 
the conjugate class $\left[\mathfrak{k}_{H}\right]$ is a local orbit type of the $K$-orbit $K(H)$ in $T_{o} M$. For a compact semisimple symmetric space $M=G / K$, Tamaru [Tama2] found a method to determine the Lie algebras of the isotropy subgroups of $K$ for each $K$-orbit in $T_{o} M$. We apply Tamaru's method to each symmetric R-space in Section 3 to get the main results.

The organization of this paper is as follows. In Section 1, we give preliminaries for the restricted root systems $\Delta$ of a compact semisimple symmetric space $M=G / K$ (cf. [Hel]). And we describe the Tamaru's method. In Section 2, we give the proof of Theorem 0.1 (Theorem 2.6), and mention that representatives of any $K$-orbit can be choosen to be a sum of elements of the positive Weyl chamber (Theorem 2.7 due to Tamaru [Tama1]). Finally, we give the proof of Corollary 0.2 (Corollary 2.8). In Subsection 2.3, we give an "algorithm" to get the isotropy subalgebras of any $K$-orbit in $T_{o} M$, which is used throughout Section 3. This paper is a part of the author's master thesis, Sophia University.

ACKNOWLEDGEMENTs. The author would like to thank Professor Soji Kaneyuki for his valuable suggestions and kind encouragements. The author is also grateful to Professor Hiroshi Tamaru for many discussions that proved very helpful to completing this work.

\section{Preliminaries}

Let $(G, K)$ be a compact semisimple symmetric pair. Let $\mathfrak{g}$ and $\mathfrak{k}$ denote the Lie algebras of $G$ and $K$ respectively. There exists the involutive automorphism $\sigma$ of $\mathfrak{g}$ such that $\mathfrak{k}$ is the $(+1)$-eigenspace of $\sigma$. Let $\mathfrak{m}$ be the (-1)-eigenspace of $\sigma$. Then we have the decomposition $\mathfrak{g}=\mathfrak{k} \oplus \mathfrak{m}$. Let $\mathfrak{a}$ be a maximal abelian subspace in $\mathfrak{m}$, which is uniquely determined up to conjugacy. We call dim $\mathfrak{a}$ the rank of the symmetric space $M:=G / K$. Let $\alpha$ be a linear form on $\mathfrak{a}$, and $\mathfrak{g}(\alpha):=\left\{X \in \mathfrak{g} \mid[H,[H, X]]=-\alpha(H)^{2} X\right.$ for any $\left.H \in \mathfrak{a}\right\}$. A non-zero linear form $\alpha$ is said to be a root, if $\mathfrak{g}(\alpha) \neq 0$. Remark that $\mathfrak{g}(\alpha)=\mathfrak{g}(-\alpha)$. Let $\mathfrak{k}(\alpha):=\mathfrak{k} \cap \mathfrak{g}(\alpha)$ and $\mathfrak{m}(\alpha):=\mathfrak{m} \cap \mathfrak{g}(\alpha)$. Observe that $\mathfrak{m}(0)=\mathfrak{a}$. The subalgebra $\mathfrak{k}(0)$ is called the pricipal isotropy subalgebra of $\mathfrak{k}$. In fact, $\mathfrak{k}(0)$ coincides with the Lie algebra of the principal isotropy subgroup of $s$-representation. We call $\operatorname{dim} \mathfrak{k}(\alpha)$ the multiplicity of a root $\alpha$. The set of all roots with the multiplicities is called the restricted root system of the symmetric space. The restricted Dynkin diagram is given by the inner product of elements of the restricted root system. Let $\Delta$ denote the restricted root system of the compact irreducible symmetric pair $(\mathfrak{g}, \mathfrak{k})$ with respect to $\mathfrak{a}$. Then we have the following decompositions, which are called the root space decompositions: $\mathfrak{k}=\mathfrak{k}(0) \oplus \sum_{\alpha \in \Delta} \mathfrak{k}(\alpha)$, and $\mathfrak{m}=\mathfrak{m}(0) \oplus \sum_{\alpha \in \Delta} \mathfrak{m}(\alpha)$. For each pair $(\mathfrak{g}, \mathfrak{k})$, Tamaru gave the table of corresponding Dinkin diagram (with multiplicities) and the principal isotropy subalgebra $\mathfrak{k}(0)$ of $\mathfrak{k}$. See Table 1 of [Tama2]. A subset $\Delta^{\prime}$ in a root system $\Delta$ is called a closed subsystem, if the following two properties hold: (i) if $\alpha, \beta \in \Delta^{\prime}$ and $\alpha+\beta \in \Delta$, then $\alpha+\beta \in \Delta^{\prime}$, (ii) $\Delta^{\prime}=-\Delta^{\prime}$. We can easily see that every closed subsystem is a root system. Furthermore, it is the restricted root system of certain symmetric space. We denote $\mathfrak{k}(0)$ the principal isotropy subalgebra. 
THEOREM 1.1 (Tamaru [Tama2]). Let $\Delta^{\prime}$ be a closed subsystem. Then, (i) there exists a compact semisimple symmetric pair $\left(\mathfrak{g}^{\prime}, \mathfrak{k}^{\prime}\right)$ whose restricted root system is $\Delta^{\prime}$, and (ii) the principal isotropy subalgebra of the pair $\left(\mathfrak{g}^{\prime}, \mathfrak{k}^{\prime}\right)$ is an ideal of the principal isotropy subalgebra $\mathfrak{k}(0)$ of the pair $(\mathfrak{g}, \mathfrak{k})$.

Tamaru [Tama2] considered the following subalgebra of the closed subsystem in the restricred root system of the pair $(\mathfrak{g}, \mathfrak{k})$; For a closed subsystem $\Delta^{\prime}$ in $\Delta$, we call $\mathfrak{k}\left(\Delta^{\prime}\right):=$ $\mathfrak{k}(0) \oplus \sum_{\alpha \in \Delta^{\prime}} \mathfrak{k}(\alpha)$ the $\Delta^{\prime}$-subalgebra. By the above theorem, there exists the compact semisimple symmetric pair $\left(\mathfrak{g}^{\prime}, \mathfrak{k}^{\prime}\right)$ whose restricted root system is $\Delta^{\prime}$. Let $\mathfrak{k}^{\prime}(0)$ denote the principal isotropy subalgebra of that pair $\left(\mathfrak{g}^{\prime}, \mathfrak{k}^{\prime}\right)$.

THEOREM 1.2 (Tamaru [Tama2]). $\quad \mathfrak{k}\left(\Delta^{\prime}\right)=\left(\mathfrak{k}(0) / \mathfrak{k}^{\prime}(0)\right) \oplus \mathfrak{k}^{\prime}$, where $\left(\mathfrak{k}(0) / \mathfrak{k}^{\prime}(0)\right)$ means the orthogonal complement of $\mathfrak{k}^{\prime}(0)$ in $\mathfrak{k}(0)$.

\section{Representatives of $K$-orbit}

2.1. The isotropy subalgebra of $S$-representation. Let $(\mathfrak{g}, \mathfrak{k})$ be a compact semisimple symmetric pair, and let $\Delta$ be the restricted root system of the pair $(\mathfrak{g}, \mathfrak{k})$ with respect to a.

Proposition 2.1. For given $H \in \mathfrak{a}$, let $\Delta_{H}:=\{\alpha \in \Delta \mid \alpha(H)=0\}$, and $\mathfrak{k}_{H}=$ $\{X \in \mathfrak{k} \mid[X, H]=0\}$. Then, (i) $\Delta_{H}$ is the closed subsystem, and (ii) $\mathfrak{k}_{H}=\mathfrak{k}\left(\Delta_{H}\right)$ is the isotropy subalgebra of the s-representation at $H \in \mathfrak{a}$, i.e., the $\Delta_{H}$-subalgebra.

Proof. By the definition, $\Delta_{H}:=\{\alpha \in \Delta \mid \alpha(H)=0\}$ is the closed subsystem of $\Delta$. On the other hand, by the definition of the root, we have the following relation such that $\mathfrak{k}_{H}=\mathfrak{k}(0) \oplus \sum_{\alpha(H) \in \Delta_{H}} \mathfrak{k}(\alpha)$.

We define $\mathfrak{g}^{H}$ and $\mathfrak{k}^{H}$ corresponding to the compact semisimple symmetric pair $\left(\mathfrak{g}^{\prime}, \mathfrak{k}^{\prime}\right)$ in Theorem 1.1 as follows. Let $\mathfrak{k}^{H}(0):=\sum_{\alpha \in \Delta_{H}}[\mathfrak{k}(\alpha), \mathfrak{k}(\alpha)]_{\mathfrak{k}(0)}$, where the subscript $\mathfrak{k}(0)$ denote the $\mathfrak{k}(0)$-component, and let $\mathfrak{a}^{H}$ be the subspace in $\mathfrak{a}$ spaned by $\Delta_{H}, \mathfrak{k}^{H}:=\mathfrak{k}^{H}(0) \oplus$ $\sum_{\alpha \in \Delta_{H}} \mathfrak{k}(\alpha), \mathfrak{m}^{H}:=\mathfrak{a}^{H} \oplus \sum_{\alpha \in \Delta_{H}} \mathfrak{m}(\alpha)$, and $\mathfrak{g}^{H}:=\mathfrak{k}^{H} \oplus \mathfrak{m}^{H}$. Then, we have the corollary to Theorem 1.1 and Theorem 1.2;

Corolary 2.2. Let $\Delta_{H}=\{\alpha \in \Delta \mid \alpha(H)=0\}$, i.e., the closed subsystem. Then, (i) $\left(\mathfrak{g}^{H}, \mathfrak{k}^{H}\right)$ is a compact semisimple symmetric pair whose restricted root system is $\Delta_{H}$, (ii) the principal isotropy subalgebra $\mathfrak{k}^{H}(0)$ of the pair $\left(\mathfrak{g}^{H}, \mathfrak{k}^{H}\right)$ is an ideal of $\mathfrak{k}(0)$, and (iii) $\mathfrak{k}_{H}=\left(\mathfrak{k}(0) / \mathfrak{k}^{H}(0)\right) \oplus \mathfrak{k}^{H}$, where $\left(\mathfrak{k}(0) / \mathfrak{k}^{H}(0)\right)$ means the orthogonal complement of $\mathfrak{k}^{H}(0)$ in $\mathfrak{k}(0)$.

2.2. Representatives of $K$-orbit. We give a criterion for the same local orbit types (Theorem 2.6). We will find good representatives of the linear isotropy orbit (Theorem 2.7). Finally, we give an evaluation of the number of the local orbit types (Corollary 2.8). 
LEMMA 2.3. Let $\left\{\alpha_{1}, \cdots, \alpha_{r}\right\}$ be a set of all simple roots of $\Delta$, where $r$ is the rank. Let $H:=\sum_{i=1}^{k} \varpi_{i}$, where $\Omega:=\left\{\varpi_{1}, \cdots, \varpi_{r}\right\}$ is the set of all fundamental weights. Then, $\left\{\alpha_{k+1}, \cdots, \alpha_{r}\right\}$ is a set of all simple roots of $\Delta_{H}$.

PROOF. Let $\alpha=\sum_{j=1}^{r} c_{j} \alpha_{j} \in \Delta_{H}$, where all $c_{j}$ are non-positive or non-negative integers. Since $0=\alpha(H)=\frac{1}{2} \sum_{i=1}^{k} c_{i}\left(\alpha_{i}, \alpha_{i}\right)$, we have $c_{1}=\cdots=c_{k}=0$. Then, $\alpha$ can be expressed as the linear combination of $\left\{\alpha_{k+1}, \cdots, \alpha_{r}\right\}$ with non-positive or non-negative coefficients.

COROLlaRY 2.4. Let $\Pi$ be a simple root system of $\Delta$, and let $\Pi^{\prime}$ be a subset of $\Pi$. Let $\Omega=\left\{\varpi_{1}, \cdots, \varpi_{r}\right\}$ be the set of all fundamental weights, where $r$ is the rank. Then, there exists a unique element $H=\sum_{l=1}^{k} \varpi_{i_{l}}$ such that $\Pi^{\prime}=\Pi_{H}$ satisfying the following conditions: (i) if $\alpha_{i} \in \Pi^{\prime}, \alpha_{i}(H)=0$ (ii) if $\alpha_{i} \notin \Pi^{\prime}, \alpha_{i}(H)=\frac{1}{2}\left(\alpha_{i}, \alpha_{i}\right)$, so that, $H=$ $\sum_{\alpha_{i} \notin \Pi^{\prime}} \varpi_{i}$.

LEMMA 2.5. Let $N_{K}(\mathfrak{a}):=\{g \in K \mid \operatorname{Ad}(g) \mathfrak{a}=\mathfrak{a}\}$ be a normalizer of $\mathfrak{a}$ in $K$, and $\Omega=\left\{\varpi_{1}, \cdots, \varpi_{r}\right\}$ be the set of all fundamental weights, where $r$ is the rank, and $\Pi_{H}$ and $\Pi_{H^{\prime}}$ be simple root systems of $\Delta_{H}$ and $\Delta_{H^{\prime}}$ respectively. Let $H:=\sum_{j=1}^{k} \varpi_{j}, H^{\prime}:=$ $\sum_{s=1}^{t} \varpi_{s}$. For given $g \in N_{K}(\mathfrak{a}), g \Delta_{H}=\Delta_{H^{\prime}}$ if and only if $g^{\prime} \Pi_{H}=\Pi_{H^{\prime}}$ for a certain $g^{\prime} \in N_{K}(\mathfrak{a})$.

PRoOF. If $g \Delta_{H}=\Delta_{H^{\prime}}$ is satisfied, we have $g \Pi_{H} \subset \Delta_{H^{\prime}}$. Then, there exists an element $w$ of Weyl groups $W\left(\Delta_{H^{\prime}}\right)$ of $\Delta_{H^{\prime}}$ such that $w\left(g \Pi_{H}\right)=\Pi_{H^{\prime}}$. Conversely, if $g^{\prime} \Pi_{H}=\Pi_{H^{\prime}}$ for a certain $g^{\prime} \in N_{K}(\mathfrak{a})$, one can easily see the assertion, by identifying $\alpha_{j} \in \Pi_{H}$ with $\varpi_{j}$ and doing $\alpha_{s} \in \Pi_{H^{\prime}}$ with $\varpi_{s}$.

THEOREM 2.6. Let $\mathcal{C}:=\left\{\sum_{i=1}^{r} c_{i} \varpi_{i} \mid c_{i} \geq 0\right.$ for $\left.i=1, \cdots, r\right\}$, where each $\varpi_{i}$ is an element of the set of all fundamental weights $\Omega=\left\{\varpi_{1}, \cdots, \varpi_{r}\right\}$. For any $H, H^{\prime} \in \mathcal{C}$, $\Delta_{H}$ is equivalent to $\Delta_{H^{\prime}}$ if and only if $\mathfrak{k}_{H}$ is conjugate to $\mathfrak{k}_{H^{\prime}}$, i.e., for given $g \in N_{K}(\mathfrak{a})$, $g \Delta_{H}=\Delta_{H^{\prime}}$ if and only if $\operatorname{Ad}(g) \mathfrak{k}_{H}=\mathfrak{k}_{H^{\prime}}$.

Proof. For given $g \in N_{K}(\mathfrak{a})$, put $g: \mathfrak{a}^{*} \rightarrow \mathfrak{a}^{*}$ such that $\alpha \mapsto g \cdot \alpha:=\alpha \circ \operatorname{Ad}\left(g^{-1}\right)$, where $\mathfrak{a}^{*}$ is a dual space of $\mathfrak{a}$. Then, we claim first that $\operatorname{Ad}(g) \mathfrak{k}(\alpha)=\mathfrak{k}(g \cdot \alpha)$. Indeed, since $\operatorname{Ad}(g) \mathfrak{k}(\alpha)=\mathfrak{k} \cap \operatorname{Ad}(g) \mathfrak{g}(\alpha)$, it suffices to observe that $\operatorname{Ad}(g) \mathfrak{g}(\alpha)=\mathfrak{g}(g \cdot \alpha)$. For any $X_{\alpha} \in$ $\mathfrak{g}(\alpha)$ and any $H \in \mathfrak{a}$, we have $\left[H,\left[H, \operatorname{Ad}(g) X_{\alpha}\right]\right]=-g \cdot \alpha(H)^{2} \operatorname{Ad}(g) X_{\alpha}$, which implies that $\operatorname{Ad}(g) X_{\alpha} \in \mathfrak{g}(g \cdot \alpha)$. This proves the first assertion. Let $\mathfrak{k}_{H}:=\mathfrak{k}(0) \oplus \sum_{\alpha \in \Delta_{H}} \mathfrak{k}(\alpha)$, and $\mathfrak{k}_{H^{\prime}}:=\mathfrak{k}(0) \oplus \sum_{\beta \in \Delta_{H}^{\prime}} \mathfrak{k}(\beta)$. If $g \Delta_{H}=\Delta_{H^{\prime}}$ is satisfied, we have $\operatorname{Ad}(g) \mathfrak{k}_{H}=\mathfrak{k}_{H^{\prime}}$. Conversely, if $\operatorname{Ad}(g) \mathfrak{k}_{H}=\mathfrak{k}_{H^{\prime}}$ is satisfied, we have $g \Delta_{H}=\Delta_{H^{\prime}}$. Indeed, we have $\operatorname{Ad}(g) \mathfrak{k}_{H}=\mathfrak{k}(0) \oplus$ $\sum_{g \cdot \alpha \in \Delta_{H}} \mathfrak{k}(g \cdot \alpha)$ and $\mathfrak{k}_{H^{\prime}}=\mathfrak{k}(0) \oplus \sum_{\beta \in \Delta_{H^{\prime}}} \mathfrak{k}(\beta)$. By hypothesis, we have $\sum_{g \cdot \alpha \in \Delta_{H}} \mathfrak{k}(g \cdot$ $\alpha)=\sum_{\beta \in \Delta_{H^{\prime}}} \mathfrak{k}(\beta)$. Suppose that $g \cdot \alpha \notin \Delta_{H}$ for all $\alpha \in \Delta_{H}$. Then, we have $\mathfrak{k}(g \cdot \alpha) \not \subset$ $\sum_{\beta \in \Delta_{H^{\prime}}} \mathfrak{k}(\beta)$. This contradicts with $\sum_{g \cdot \alpha \in \Delta_{H}} \mathfrak{k}(g \cdot \alpha)=\sum_{\beta \in \Delta_{H^{\prime}}} \mathfrak{k}(\beta)$. This proves that 
$g \Delta_{H} \subset \Delta_{H^{\prime}}$. One can easily see that $g \Delta_{H} \supset \Delta_{H^{\prime}}$ in a similar way. It follows that $g \Delta_{H}=$ $\Delta_{H^{\prime}}$.

In [Tama1], Tamaru showed the following;

THEOREM 2.7 (Tamaru [Tama1]). Let $\Omega=\left\{\varpi_{1}, \cdots, \varpi_{r}\right\}$ be the set of all fundamental weights, where $r$ is the rank. For any vector $H \in \mathfrak{m}, \mathfrak{k}_{H}$ is conjugate to the isotropy subalgebra at $\sum_{j=1}^{k} \varpi_{i_{j}}$, where $0 \leq k \leq r$.

COROLLARY 2.8. The number of the local orbit types of s-representations of a compact semisimple symmetric space is less than or equal to $2^{r}$, where $r$ is the rank of the symmetric space.

Proof of Corollary 2.8. By Theorem 2.7, how to choose $\left\{\varpi_{i_{j}}\right\}_{1 \leq j \leq k}$ out of $\Omega=\left\{\varpi_{1}, \cdots, \varpi_{r}\right\}$ is ${ }_{r} C_{0}+{ }_{r} C_{1}+\cdots+{ }_{r} C_{r}=(1+1)^{r}=2^{r}$. But, by Theorem 2.6, the number of the local orbit types is less than $2^{r}$.

2.3. The algorithm to get the isotropy subalgebras. The method to get the isotropy subalgebras $\mathfrak{k}_{H}$ of $\mathfrak{k}$ at $H$ is as follows : Let $(\mathfrak{g}, \mathfrak{k})$ be a compact semisimple symmetric pair. Then,

(Algorithm 1) By inspecting Tamaru's Table 1, we find the pair $(\mathfrak{g}, \mathfrak{k})$ to get the corresponding type, rank, and multiplicities. Then, we know the restricted Dynkin diagram, and the principal isotropy subalgebra $\mathfrak{k}(0)$ also. For example, in the case of $(\mathfrak{g}, \mathfrak{k})=A I_{3}=$ $(s u(4), s o(4))$, the Dynkin diagram of the pair $(s u(4), s o(4))$ is $o^{1}-o^{1}-o^{1}$, where the number on each vertex is the multiplicity, and the principal isotropy subalgebra $\mathfrak{k}(0)$ of the pair $(s u(4), s o(4))$ is 0 , by $A(1)_{3}$-type of Tamaru's Table 1.

(Algorithm 2) By Corollary 2.4, we can list all subset $\Pi^{\prime}=\Pi_{H} \subset \Pi$ corresponding to all possible subdiagrams of the Dynkin diagram obtained in Algorithm 1. And, by Corollary 2.4 , we can easily determine each $H$ from the Dynkin diagrams of each $\Pi_{H}$. In the case of the above example, the Dynkin diagrams of each $\Pi_{H}$ are (1) $\circ^{1}-\circ^{1}-\circ^{1}$, (2) $\circ^{1}-\circ^{1}$, (3) $\circ^{1} o^{1},(4) \circ^{1}$, and (5) $\{\emptyset\}$, by the Dynkin diagram $\circ^{1}-o^{1}-\circ^{1}$ of the pair $(s u(4)$, so(4)). Thus, each $H$ are $H=\{0\}$ for (1), $H=\varpi_{1}$, or $\varpi_{3}$ for (2), $H=\varpi_{2}$ for (3), $H=\varpi_{1}+\varpi_{2}$, $\varpi_{2}+\varpi_{3}$ or $\varpi_{1}+\varpi_{3}$ for (4), and $H=\sum_{i=1}^{3} \varpi_{i}$ for (5).

(Algorithm 3) From the Dynkin subdiagram corresponding to each subset $\Pi^{\prime}=\Pi_{H}$ of $\Pi$, we find the corresponding symmetric pair $\left(\mathfrak{g}^{H}, \mathfrak{k}^{H}\right)$, and the principal isotropy subalgebra $\mathfrak{k}^{H}(0)$, by using Tamaru's Table 1 . For the case of (2) of the above example, $\left(\mathfrak{g}^{H}, \mathfrak{k}^{H}\right)$ and $\mathfrak{k}^{H}(0)$ are $(s u(3), s o(3))$ and 0 , respectively, by $A(1)_{2}$-type of Tamaru's Table 1 . For the other cases, one can get explicitly each $\left(\mathfrak{g}^{H}, \mathfrak{k}^{H}\right)$ in the same way.

(Algorithm 4) By Corollary 2.2, we can determine explicitly isotropy subalgebras of each $\Pi_{H}$, i.e., $\mathfrak{k}_{H}=\left(\mathfrak{k}(0) / \mathfrak{k}^{H}(0)\right) \oplus \mathfrak{k}^{H}$. For the case of (2) of the above example, $\mathfrak{k}_{\varpi_{1}}=$ $\mathfrak{k}_{\varpi_{3}}=(0 / 0) \oplus \operatorname{so}(3)=s o(3)$. Here, $\mathfrak{k}_{\varpi_{1}}=\mathfrak{k}_{\varpi_{3}}$ is true by Theorem 2.6. In addition, the 
number of the isotropy subalgebras of $s$-representations of $A I_{3}$ is 5 , i.e., less than $2^{3}$. This fact is suitable to Corollary 2.8 .

\section{The classification of local orbit types of $S$-representations}

Let $M$ be a compact symmetric space, and let $G$ be the identity component of the isometry group of $M$. We say that $M$ is a symmetric $R$-space iff there exists a subgroup $L \subset \operatorname{Diff}(M)$ such that $L$ is the maximal compact semisimple subgroup of $G$. Now, let $K$ be the isotropy subgroup of $G$ at the origin $o \in M$. Thus, we have the symmetric R-space $M=G / K$. Let $K_{H}$ be the isotropy subgroup of $K$ (identified with the linear isotropy group) at $H$ in the tangent space $T_{o} M$. We denote by $\mathfrak{k}_{H}$ the Lie algebra of $K_{H}$. Let $\Delta$ be the restricted root system of symmetric R-space $M=G / K$, and $\Pi$ be the simple root system of $\Delta$. We denote by $\Delta_{H} \subset \Delta$ the closed subsystem. Thus, we have the simple root system $\Pi_{H}:=\Pi \cap \Delta_{H}$ of $\Delta_{H}$. Throughout this section, we obeyed the Algorithm in Subsection 2.3. One can easily give the proofs of the following results in a similar way, so we omitted the proof.

3.1. The classification of local orbit types $\left[\mathfrak{k}_{H}\right]$ of $S$-representations of symmetric R-spaces of classical types of the rank 2

$\left(T \cdot A I_{2}=U(3) / O(3)\right)$

\begin{tabular}{c|c|c|c|c|c|c}
\hline & $\Pi_{H}$ & $H$ & $\mathfrak{k}_{H}$ & $K / K_{H}$ & $\operatorname{dim} K / K_{H}$ & \\
\hline$(1)$ & $\circ^{1}-\circ^{1}$ & $\{o\}$ & $s o(3)$ & $\{o\}$ & 0 & \\
\hline$(2)$ & $\circ^{1}$ & $\varpi_{1}\left(\varpi_{2}\right)$ & $s o(2)$ & $S^{2}$ & 2 & Symmetric space \\
\hline$(3)$ & $\emptyset$ & $\varpi_{1}+\varpi_{2}$ & 0 & $S O(3)$ & 3 & Symmetric R-space \\
\hline
\end{tabular}

$\left(T \cdot A I_{2}=U(6) / S p(3)\right)$

\begin{tabular}{c|c|c|c|c|c|c}
\hline & $\Pi_{H}$ & $H$ & $\mathfrak{k}_{H}$ & $K / K_{H}$ & $\operatorname{dim} K / K_{H}$ & \\
\hline$(1)$ & $\circ^{4}-\circ^{4}$ & $\{o\}$ & $s p(3)$ & $\{o\}$ & 0 & \\
\hline$(2)$ & $\circ^{4}$ & $\varpi_{1}\left(\varpi_{2}\right)$ & $s p(1) \oplus s p(2)$ & $P^{2}(\mathbf{H})$ & 8 & Symmetric R-space \\
\hline$(3)$ & $\emptyset$ & $\varpi_{1}+\varpi_{2}$ & $s p(1)^{3}$ & $\frac{S p(3)}{S p(1)^{3}}$ & 12 & $P^{1}(\mathbf{H})$-bundle over $P^{2}(\mathbf{H})$ \\
\hline
\end{tabular}

$\left(A I I I_{2}=G r_{2}\left(\mathbf{C}^{2+q}\right)=S U(2+q) / S(U(2) \times U(q)), q \geq 2\right)$

\begin{tabular}{c|c|c|c|c|c|c}
\hline & $\Pi_{H}$ & $H$ & $\mathfrak{k}_{H}$ & $K / K_{H}$ & $\operatorname{dim} K / K_{H}$ & \\
\hline$(1)$ & $\circ^{2} \Longleftrightarrow \odot^{2(q-2), 1}$ & $\{o\}$ & $s u(2) \oplus s u(q) \oplus \mathbf{R}$ & $\{o\}$ & 0 & \\
\hline$(2)$ & $\circ^{2}$ & $\varpi_{2}$ & $u(q-2) \oplus s o(3)$ & $\frac{U(q)}{U(q-2)}$ & $4 q-4$ & Stiefel manifold \\
\hline$(3)$ & $\odot^{2(q-2), 1}$ & $\varpi_{1}$ & $\mathbf{R} \oplus u(q-1)$ & $S^{2} \times S^{2 q-1}$ & $2 q+1$ & Symmetric space \\
\hline$(4)$ & $\emptyset$ & $\varpi_{1}+\varpi_{2}$ & $\mathbf{R} \oplus u(q-2)$ & $S^{2} \times \frac{U(q)}{U(q-2)}$ & $4 q-2$ & $S^{2}$-bundle over (2) \\
\hline
\end{tabular}


$\left(B D I_{2}=S O(q+2) / S O(q) \times S O(2), q \geq 2\right)$

\begin{tabular}{c|c|c|c|c|c|c}
\hline & $\Pi_{H}$ & $H$ & $\mathfrak{k}_{H}$ & $K / K_{H}$ & $\operatorname{dim} K / K_{H}$ & \\
\hline$(1)$ & $\circ^{1} \Longrightarrow{ }^{q-2}$ & $\{o\}$ & $s o(2) \oplus s o(q)$ & $\{o\}$ & 0 & \\
\hline$(2)$ & ${ }^{q-2}$ & $\varpi_{1}$ & $s o(q-1)$ & $S^{1} \times S^{q-1}$ & $q$ & Symmetric space \\
\hline$(3)$ & $0^{1}$ & $\varpi_{2}$ & $s o(2) \oplus \operatorname{so}(q-2)$ & $\frac{S O(q)}{S O(q-2)}$ & $2 q-3$ & Stiefel manifold \\
\hline$(4)$ & $\emptyset$ & $\varpi_{1}+\varpi_{2}$ & $s o(q-2)$ & $S^{1} \times \frac{S O(q)}{S O(q-2)}$ & $2 q-2$ & $S^{1}$-bundle over (3) \\
\hline
\end{tabular}

$\left(C I_{2}=S p(2) / U(2)\right)$

\begin{tabular}{c|c|c|c|c|c|c}
\hline & $\Pi_{H}$ & $H$ & $\mathfrak{k}_{H}$ & $K / K_{H}$ & $\operatorname{dim} K / K_{H}$ & \\
\hline$(1)$ & $0^{1} \Longleftarrow \circ^{1}$ & $\{o\}$ & $u(2)$ & $\{o\}$ & 0 & \\
\hline$(2)$ & $0^{1}$ & $\varpi_{1}\left(\varpi_{2}\right)$ & $u(1)$ & $S^{3}$ & 3 & Symmetric R-space \\
\hline$(3)$ & $\emptyset$ & $\varpi_{1}+\varpi_{2}$ & 0 & $U(2)$ & 4 & Symmetric R-space \\
\hline
\end{tabular}

$\left(C I_{2}=G r_{2}\left(\mathbf{H}^{2+q}\right)=S p(2+q) / S p(2) \times S p(q), q \geq 2\right)$

\begin{tabular}{c|c|c|c|c|c|c}
\hline & $\Pi_{H}$ & $H$ & $\mathfrak{k}_{H}$ & $K / K_{H}$ & $\operatorname{dim} K / K_{H}$ & \\
\hline$(1)$ & $\circ^{4} \Longleftrightarrow \odot 4(q-2), 3$ & $\{o\}$ & $s p(2) \oplus s p(q)$ & $\{o\}$ & 0 & \\
\hline$(2)$ & $\circ^{4}$ & $\varpi_{2}$ & $s p(q-2) \oplus s o(5)$ & $\frac{S p(q)}{S p(q-2)}$ & $8 q-6$ & Stiefel manifold \\
\hline$(3)$ & $\odot^{4(q-2), 3}$ & $\varpi_{1}$ & $s p(1)^{2} \oplus s p(q-1)$ & $S^{4} \times S^{4 q-1}$ & $4 q+3$ & Symmetric space \\
\hline$(4)$ & $\varnothing$ & $\varpi_{1}+\varpi_{2}$ & $s p(1)^{2} \oplus s p(q-2)$ & $S^{4} \times \frac{S p(q)}{S p(q-2)}$ & $8 q-2$ & $S^{4}$-bundle over (2) \\
\hline
\end{tabular}

$\left(C I_{2}=G r_{2}\left(\mathbf{H}^{4}\right)=S p(4) / S p(2) \times S p(2)\right)$

\begin{tabular}{c|c|c|c|c|c|c}
\hline & $\Pi_{H}$ & $H$ & $\mathfrak{k}_{H}$ & $K / K_{H}$ & $\operatorname{dim} K / K_{H}$ & \\
\hline$(1)$ & $\circ^{4} \Longleftarrow \circ^{3}$ & $\{o\}$ & $s p(2) \oplus \operatorname{sp}(2)$ & $\{o\}$ & 0 & \\
\hline$(2)$ & $\circ^{4}$ & $\varpi_{2}$ & $s o(5)$ & $S O(5)$ & 10 & Symmetric R-space \\
\hline$(3)$ & $\circ^{3}$ & $\varpi_{1}$ & $s p(1) \oplus s o(4)$ & $S^{7} \times S^{4}$ & 11 & Symmetric space \\
\hline$(4)$ & $\varnothing$ & $\varpi_{1}+\varpi_{2}$ & $s p(1)^{2}$ & $S^{7} \times S^{7}$ & 14 & Symmetric space \\
\hline
\end{tabular}


$\left(D I_{2}=S O(8) / U(4)\right)$

\begin{tabular}{c|c|c|c|c|c|c}
\hline & $\Pi_{H}$ & $H$ & $\mathfrak{k}_{H}$ & $K / K_{H}$ & $\operatorname{dim} K / K_{H}$ & \\
\hline$(1)$ & $0^{4} \Longleftarrow \circ^{1}$ & $\{o\}$ & $u(4)$ & $\{o\}$ & 0 & \\
\hline$(2)$ & $0^{4}$ & $\varpi_{2}$ & $s o(5)$ & $S^{1} \times S^{5}$ & 6 & Symmetric space \\
\hline$(3)$ & $0^{1}$ & $\varpi_{1}$ & $s u(2)^{2} \oplus \operatorname{so}(2)$ & $\frac{S O(6)}{S O(3) \times S O(3)}$ & 9 & Symmetric R-space \\
\hline$(4)$ & $\emptyset$ & $\varpi_{1}+\varpi_{2}$ & $s p(1)^{2}$ & $\frac{U(4)}{S p(1)^{2}}$ & 10 & $S^{1}$-bundle over (3) \\
\hline
\end{tabular}

$\left(D I I I_{2}=S O(10) / U(5)\right)$

\begin{tabular}{c|c|c|c|c|c|c}
\hline & $\Pi_{H}$ & $H$ & $\mathfrak{k}_{H}$ & $K / K_{H}$ & $\operatorname{dim} K / K_{H}$ & \\
\hline$(1)$ & $\circ^{4} \Longleftrightarrow \odot \odot^{4,1}$ & $\{o\}$ & $u(5)$ & $\{o\}$ & 0 & \\
\hline$(2)$ & $\odot^{4,1}$ & $\varpi_{1}$ & $s p(1) \oplus u(3)$ & $\frac{S U(5)}{S U(2) \times S U(3)}$ & 13 & Symmetric R-space \\
\hline$(3)$ & $\circ^{4}$ & $\varpi_{2}$ & $\mathbf{R} \oplus s o(5)$ & $\frac{S U(5)}{S O(5)}$ & 14 & Symmetric R-space \\
\hline$(4)$ & $\varnothing$ & $\varpi_{1}+\varpi_{2}$ & $s u(2)^{2} \oplus \mathbf{R}$ & $\frac{S U(5)}{S U(2) \times S U(2)}$ & 18 & $\begin{array}{c}\text { Flag manifold } \\
\left(S^{4} \text {-bundle over (2)) }\right.\end{array}$ \\
\hline
\end{tabular}

3.2. The classification of local orbit types $\left[\mathfrak{k}_{H}\right]$ of $S$-representations of symmetric R-spaces of classical types of the rank 3

$\left(T \cdot A I_{3}=U(4) / O(4)\right)$

\begin{tabular}{c|c|c|c|c|c|c}
\hline & $\Pi_{H}$ & $H$ & $\mathfrak{k}_{H}$ & $K / K_{H}$ & $\operatorname{dim} K / K_{H}$ & \\
\hline$(1)$ & $\circ^{1}-\circ^{1}-\circ^{1}$ & $\{o\}$ & $s o(4)$ & $\{o\}$ & 0 & \\
\hline$(2)$ & $\circ^{1}-\circ^{1}$ & $\varpi_{1}\left(\varpi_{3}\right)$ & $s o(3)$ & $S^{3}$ & 3 & Symmetric R-space \\
\hline$(3)$ & $\circ^{1} \circ^{1}$ & $\varpi_{2}$ & $s o(2)^{2}$ & $\frac{S O(4)}{S O(2) \times S O(2)}$ & 4 & Symmetric R-space \\
\hline$(4)$ & $\circ^{1}$ & $\varpi_{i}+\varpi_{j}(i \neq j)$ & $s o(2)$ & $\frac{S O(4)}{S O(2)}$ & 5 & Stiefel manifold \\
\hline$(5)$ & $\varnothing$ & $\varpi_{1}+\varpi_{2}+\varpi_{3}$ & 0 & $S O(4)$ & 6 & Symmetric R-space \\
\hline
\end{tabular}


$\left(T \cdot A I_{3}=S U(8) / S p(4) \mathbf{Z}_{2}\right)$

\begin{tabular}{c|c|c|c|c|c|c}
\hline & $\Pi_{H}$ & $H$ & $\mathfrak{k}_{H}$ & $K / K_{H}$ & $\operatorname{dim} K / K_{H}$ & \\
\hline$(1)$ & $\circ^{4}-\circ^{4}-\circ^{4}$ & $\{o\}$ & $s p(4)$ & $\{o\}$ & 0 & \\
\hline$(2)$ & $\circ^{4}-\circ^{4}$ & $\varpi_{1}\left(\varpi_{3}\right)$ & $s p(1) \oplus \operatorname{sp}(3)$ & $P^{3}\left(\mathbf{H}^{4}\right)$ & 12 & Symmetric R-space \\
\hline$(3)$ & $\circ^{4} \circ^{4}$ & $\varpi_{2}$ & $s o(5) \oplus \operatorname{so}(5)$ & $G r_{2}\left(\mathbf{H}^{4}\right)$ & 16 & Symmetric R-space \\
\hline$(4)$ & $\circ^{4}$ & $\varpi_{i}+\varpi_{j}(i \neq j)$ & $s p(1)^{2} \oplus \operatorname{so}(5)$ & $\frac{S p(4)}{S p(1)^{2} \times S p(2)}$ & 20 & 2nd kind GLA \\
\hline$(5)$ & $\varnothing$ & $\varpi_{1}+\varpi_{2}+\varpi_{3}$ & $s p(1)^{4}$ & $\frac{S p(4)}{S p(1)^{4}}$ & 24 & 1st kind GLA \\
\hline
\end{tabular}

$$
\left(A I I I_{3}=G r_{3}\left(\mathbf{C}^{3+q}\right), q \geq 3\right)
$$

\begin{tabular}{|c|c|c|c|c|c|c|}
\hline & $\Pi_{H}$ & $H$ & $\mathfrak{k}_{H}$ & $K / K_{H}$ & $\operatorname{dim} K / K_{H}$ & \\
\hline (1) & $\circ^{2}-\circ^{2} \Longleftrightarrow \odot^{2(q-3), 1}$ & $\{o\}$ & $s u(3) \oplus s u(q) \oplus \mathbf{R}$ & $\{o\}$ & 0 & \\
\hline (2) & $o^{2}-o^{2}$ & $\varpi_{3}$ & $u(q-3) \oplus s u(3)$ & $\frac{S U(q)}{S U(q-3)}$ & $6 q-9$ & Stiefel manifold \\
\hline (3) & $\circ^{2} \Longleftrightarrow \odot^{2(q-3), 1}$ & $\varpi_{1}$ & $\begin{array}{c}\mathbf{R} \oplus s u(2) \oplus \\
s u(q-1) \oplus \mathbf{R}\end{array}$ & $P^{2}(\mathbf{C}) \times S^{2 q-1}$ & $2 q+3$ & Symmetric space \\
\hline (4) & $\circ^{2} \odot^{2(q-3), 1}$ & $\varpi_{2}$ & $\begin{array}{l}u(q-3) \oplus \operatorname{so}(3) \\
\oplus \operatorname{su}(q-2) \oplus \mathbf{R}\end{array}$ & $\frac{(S U(3) \times U(q))}{(U(q-3) \times S U(2) \times U(q-2))}$ & $-q^{2}+10 q-8$ & \\
\hline (5) & $0^{2}$ & $\begin{array}{c}\varpi_{1}+\varpi_{3} \\
\left(\varpi_{2}+\varpi_{3}\right)\end{array}$ & $\begin{array}{l}\mathbf{R} \oplus u(q-3) \\
\quad \oplus \operatorname{so}(3)\end{array}$ & $S^{5} \times S U(q) / U(q-3)$ & $6 q-5$ & \\
\hline (6) & $\overbrace{}^{2(q-3), 1}$ & $\varpi_{1}+\varpi_{2}$ & $\begin{array}{c}\mathbf{R} \oplus u(q-3) \\
\oplus s u(q-2) \oplus \mathbf{R}\end{array}$ & $\frac{(S U(3) \times U(q))}{(U(1) \times U(q-3) \times U(q-2))}$ & $-q^{2}+10 q-6$ & \\
\hline (7) & $\emptyset$ & $\sum_{i=1}^{3} \varpi_{i}$ & $\mathbf{R}^{2} \oplus u(q-3)$ & $\frac{(S U(3) \times S U(q))}{(U(1) \times U(q-3))}$ & $6 q-3$ & \\
\hline
\end{tabular}

$\left(B D I_{3}=G r_{3}\left(\mathbf{R}^{3+q}\right), q \geq 3\right)$

\begin{tabular}{c|c|c|c|c|c|c}
\hline & $\Pi_{H}$ & $H$ & $\mathfrak{k}_{H}$ & $K / K_{H}$ & $\operatorname{dim} K / K_{H}$ & \\
\hline$(1)$ & $\circ^{1}-\circ^{1} \Longrightarrow \circ^{(q-3)}$ & $\{o\}$ & $s o(q) \oplus \operatorname{so}(3)$ & $\{o\}$ & 0 & \\
\hline$(2)$ & $\circ^{1}-\circ^{1}$ & $\varpi_{3}$ & $s o(q-3) \oplus \operatorname{so}(3)$ & $\frac{S O(q)}{S O(q-3)}$ & $3 q-6$ & Stiefel manifold \\
\hline$(3)$ & $0^{1} \Longrightarrow \circ^{(q-3)}$ & $\varpi_{1}$ & $s o(2) \oplus \operatorname{so}(q-1)$ & $S^{q-1} \times S^{2}$ & $q+1$ & Symmetric space \\
\hline$(4)$ & $\circ^{1} \odot^{(q-3)}$ & $\varpi_{2}$ & $s o(2) \oplus \operatorname{so}(q-2)$ & $\frac{S O(q)}{S O(q-2)} \times S^{2}$ & $2 q-1$ & $\left(S^{q-3} \times S^{2}\right)$-bundle over (2) \\
\hline$(5)$ & $0^{1}$ & $\begin{array}{c}\varpi_{1}+\varpi_{3} \\
\left(\varpi_{2}+\varpi_{3}\right)\end{array}$ & $s o(q-3) \oplus \operatorname{so}(2)$ & $\frac{S O(q)}{S O(q-3)} \times S^{2}$ & $3 q-4$ & $S^{q-3}$-bundle over (4) \\
\hline$(6)$ & $\circ^{(q-3)}$ & $\varpi_{1}+\varpi_{2}$ & $s o(q-2)$ & $\frac{S O(q)}{S O(q-2)} \times S^{3}$ & $2 q$ & $S^{1}$-bundle over (4) \\
\hline$(7)$ & $\emptyset$ & $\sum_{i=1}^{3} \varpi_{i}$ & $s o(q-3)$ & $\frac{S O(q)}{S O(q-3)} \times S^{3}$ & $3 q-3$ & $S^{3}$-bundle over (2) \\
\hline
\end{tabular}


$\left(C I_{3}=S p(3) / U(3)\right)$

\begin{tabular}{c|c|c|c|c|c|c}
\hline & $\Pi_{H}$ & $H$ & $\mathfrak{k}_{H}$ & $K / K_{H}$ & $\operatorname{dim} K / K_{H}$ & \\
\hline$(1)$ & $\circ^{1}-\circ^{1} \Longleftarrow \circ^{1}$ & $\{o\}$ & $u(3)$ & $\{o\}$ & 0 & \\
\hline$(2)$ & $\circ^{1}-\circ^{1}$ & $\varpi_{3}$ & $s o(3)$ & $S^{5} \times S^{1}$ & 6 & Symmetric space \\
\hline$(3)$ & $\circ^{1} \Longleftarrow \circ^{1}$ & $\varpi_{1}$ & $u(2)$ & $S^{5}$ & 5 & Symmetric space \\
\hline$(4)$ & $\circ^{1} \circ^{1}$ & $\varpi_{2}$ & $s o(2)^{2}$ & $\frac{U(3)}{(U(1) \times U(1))}$ & 7 & Flag manifold \\
\hline$(5)$ & $\circ^{1}$ & $\begin{array}{c}\varpi_{1}+\varpi_{3} \\
\left(\varpi_{2}+\varpi_{3}\right)\end{array}$ & $s o(2)$ & $S U(3)$ & 8 & $S^{1}$-bundle over (4) \\
\hline$(6)$ & $\varnothing$ & $\sum_{i=1}^{3} \varpi_{i}$ & $\{0\}$ & $U(3)$ & 9 & Symmetric R-space \\
\hline
\end{tabular}

$$
\left(C I_{3}=G r_{3}\left(\mathbf{H}^{3+q}\right), q \geq 3\right)
$$

\begin{tabular}{c|c|c|c|c|c|c}
\hline & $\Pi_{H}$ & $H$ & $\mathfrak{k}_{H}$ & $K / K_{H}$ & $\operatorname{dim} K / K_{H}$ & \\
\hline$(1)$ & $\circ^{4}-\circ^{4} \Longleftrightarrow \odot \odot^{4(q-3), 3}$ & $\{o\}$ & $s p(3) \oplus s p(q)$ & $\{o\}$ & 0 & \\
\hline$(2)$ & $\circ^{4}-\circ^{4}$ & $\varpi_{3}$ & $s p(q-3) \oplus s p(3)$ & $\frac{S p(q)}{S p(q-3)}$ & $12 q-15$ & Stiefel manifold \\
\hline$(3)$ & $0^{4} \Longleftrightarrow \odot^{4(q-3), 3}$ & $\varpi_{1}$ & $\begin{array}{c}s p(1) \oplus s p(2) \\
\oplus p(q-1)\end{array}$ & $P^{2}(\mathbf{H}) \times S^{4 q-1}$ & $4 q+7$ & Symmetric space \\
\hline$(4)$ & $\circ^{4} \odot^{4(q-3), 3}$ & $\varpi_{2}$ & $\begin{array}{c}s p(2) \oplus s p(1) \\
\oplus s p(q-2)\end{array}$ & $P^{2}(\mathbf{H}) \times \frac{S p(q)}{S p(q-2)}$ & $8 q+2$ & $S^{4 q-5}$-bundle over (3) \\
\hline$(5)$ & $\circ^{4}$ & $\begin{array}{c}\varpi_{1}+\varpi_{3} \\
\left(\varpi_{2}+\varpi_{3}\right)\end{array}$ & $\begin{array}{c}s p(1) \oplus s p(q-3) \\
\oplus s p(2)\end{array}$ & $P^{2}(\mathbf{H}) \times \frac{S p(q)}{S p(q-3)}$ & $12 q-7$ & $S^{4 q-9}$-bundle over (4) \\
\hline$(6)$ & $\odot^{4(q-3), 3}$ & $\varpi_{1}+\varpi_{2}$ & $s p(1)^{3} \oplus s p(q-2)$ & $\frac{S p(3)}{S p(1)^{3} \times \frac{S p(q)}{S p(q-2)}}$ & $8 q+6$ & $P^{1}(\mathbf{H})$-bundle over (4) \\
\hline$(7)$ & $\emptyset$ & $\sum_{i=1}^{3} \varpi_{i}$ & $s p(1)^{3} \oplus s p(q-3)$ & $\frac{S p(3)}{S p(1)^{3}} \times \frac{S p(q)}{S p(q-3)}$ & $12 q-3$ & $S^{4 q-9}$-bundle over (4) \\
\hline
\end{tabular}

$\left(\mathrm{DIII}_{3}=S O(12) / U(6)\right)$

\begin{tabular}{c|c|c|c|c|c|c}
\hline & $\Pi_{H}$ & $H$ & $\mathfrak{k}_{H}$ & $K / K_{H}$ & $\operatorname{dim} K / K_{H}$ & \\
\hline$(1)$ & $0^{4}-\circ^{4} \Longleftarrow \circ^{1}$ & $\{o\}$ & $u(6)$ & $\{o\}$ & 0 & \\
\hline$(2)$ & $\circ^{4}-\circ^{4}$ & $\varpi_{3}$ & $s p(3)$ & $\frac{U(6)}{S p(3)}$ & 15 & Symmetric R-space \\
\hline$(3)$ & $\circ^{4} \Longleftarrow \circ^{1}$ & $\varpi_{1}$ & $s u(2) \oplus u(4)$ & $\frac{S U(6)}{S U(2) \times S U(4)}$ & 17 & \\
\hline$(4)$ & $\circ^{4} \circ^{1}$ & $\varpi_{2}$ & $s u(2) \oplus s p(2) \oplus \operatorname{so}(2)$ & $\frac{S U(6)}{S U(2) \times S p(2)}$ & 22 & $S^{5}$-bundle over (3) \\
\hline$(5)$ & $\circ^{4}$ & $\varpi_{1}+\varpi_{3}$ & $s u(2) \oplus s p(2)$ & $\frac{U(6)}{S U(2) \times S p(2)}$ & 23 & $S^{1}$-bundle over (4) \\
\hline$(6)$ & $0^{1}$ & $\varpi_{1}+\varpi_{2}$ & $s u(2)^{3} \oplus s o(2)$ & $\frac{S U(6)}{S U(2)^{3}}$ & 26 & \\
\hline$(7)$ & $\varnothing$ & $\sum_{i=1}^{3} \varpi_{i}$ & $s u(2)^{3}$ & $\frac{U(6)}{S U(2)^{3}}$ & 27 & $S^{1}$-bundle over (6) \\
\hline
\end{tabular}


$\left(D I_{3}=S O(14) / U(7)\right)$

\begin{tabular}{|c|c|c|c|c|c|}
\hline & $\Pi_{H}$ & $H$ & $\mathfrak{k}_{H}$ & $K / K_{H}$ & $\operatorname{dim} K / K_{H}$ \\
\hline (1) & $\circ^{4}-\circ^{4} \Longleftrightarrow \odot^{4,1}$ & $\{o\}$ & $u(7)$ & $\{o\}$ & 0 \\
\hline (2) & $\circ^{4} \Longleftrightarrow \odot^{4,1}$ & $\varpi_{1}$ & $s u(2) \oplus u(5)$ & $\frac{S U(7)}{S U(2) \times S U(5)}$ & 21 \\
\hline (3) & $0^{4}-o^{4}$ & $\varpi_{3}$ & $\mathbf{R} \oplus \operatorname{sp}(3)$ & $\frac{S U(7)}{S p(3)}$ & 27 \\
\hline (4) & $\circ^{4} \bigcirc^{4,1}$ & $\varpi_{2}$ & $s p(2) \oplus u(3)$ & $\frac{S U(7)}{S p(2) \times S U(3)}$ & 30 \\
\hline (5) & $\odot^{4,1}$ & $\varpi_{1}+\varpi_{2}$ & $s u(2)^{2} \oplus u(3)$ & $\frac{S U(7)}{S U(2)^{2} \times S U(3)}$ & 34 \\
\hline (6) & $0^{4}$ & $\begin{array}{c}\varpi_{1}+\varpi_{3} \\
\left(\varpi_{2}+\varpi_{3}\right)\end{array}$ & $s u(2) \oplus \mathbf{R} \oplus s p(2)$ & $\frac{S U(7)}{S U(2) \times S p(2)}$ & 35 \\
\hline (7) & $\emptyset$ & $\sum_{i=1}^{3} \varpi_{i}$ & $s u(2)^{3} \oplus \mathbf{R}$ & $\frac{S U(7)}{S U(2)^{3}}$ & 39 \\
\hline
\end{tabular}

3.3. The classification of local orbit types $\left[\mathfrak{k}_{H}\right]$ of $S$-representations of symmetric R-spaces of exceptional types

$\left(E I I I=E_{6} / \operatorname{Spin}(10) \cdot T\right)$

\begin{tabular}{c|c|c|c|c|c|c}
\hline & $\Pi_{H}$ & $H$ & $\mathfrak{k}_{H}$ & $K / K_{H}$ & $\operatorname{dim} K / K_{H}$ & \\
\hline$(1)$ & $0^{6} \Longleftrightarrow \odot^{8,1}$ & $\{o\}$ & $\operatorname{so}(2) \oplus \operatorname{so}(10)$ & $\{o\}$ & 0 & \\
\hline$(2)$ & $\odot^{8,1}$ & $\varpi_{1}$ & $\operatorname{so}(2) \oplus \operatorname{su}(5)$ & $\frac{\operatorname{Spin}(10)}{\operatorname{SU}(5)}$ & 21 & Homogeneous CR-manifold \\
\hline$(3)$ & $0^{6}$ & $\varpi_{2}$ & $\operatorname{so}(2) \oplus \operatorname{so}(7)$ & $\frac{\operatorname{Spin}(10)}{\operatorname{Spin}(7)}$ & 24 & Stiefel manifold \\
\hline$(4)$ & $\emptyset$ & $\varpi_{1}+\varpi_{2}$ & $u(4)$ & $\frac{\operatorname{Spin}(10)}{\operatorname{Spin}(6)}$ & 30 & Stiefel manifold \\
\hline
\end{tabular}

$\left(E N=T \cdot E_{6} / F_{4}\right)$

\begin{tabular}{c|c|c|c|c|c|c}
\hline & $\Pi_{H}$ & $H$ & $\mathfrak{k}_{H}$ & $K / K_{H}$ & $\operatorname{dim} K / K_{H}$ & \\
\hline$(1)$ & $\circ^{8}-\circ^{8}$ & $\{o\}$ & $f_{4}$ & $\{o\}$ & 0 & \\
\hline$(2)$ & $\circ^{8}$ & $\varpi_{i}(i=1,2)$ & $\operatorname{so}(9)$ & $P^{2}(\mathbf{O})=\frac{F_{4}}{S O(9)}$ & 16 & Symmetric R-space \\
\hline$(3)$ & $\varnothing$ & $\varpi_{1}+\varpi_{2}$ & $s o(8)$ & $\frac{F_{4}}{S O(8)}$ & 24 & $S^{8}$-bundle over $P^{2}(\mathbf{O})$ \\
\hline
\end{tabular}


$\left(E V I I=E_{7} / E_{6} \cdot T\right)$

\begin{tabular}{|c|c|c|c|c|c|c|}
\hline & $\Pi_{H}$ & $H$ & $\mathfrak{k}_{H}$ & $K / K_{H}$ & $\operatorname{dim} K / K_{H}$ & \\
\hline (1) & $o^{8}-o^{8} \Longleftarrow 0^{1}$ & $\{o\}$ & $s o(2) \oplus e_{6}$ & $\{o\}$ & 0 & \\
\hline (2) & $0^{8}-0^{8}$ & $\varpi_{3}$ & $f_{4}$ & $\frac{T \cdot E_{6}}{F_{4}}$ & 27 & Symmetric R-space \\
\hline (3) & $\circ^{8} \Longleftarrow \circ^{1}$ & $\varpi_{1}$ & $s o(2) \oplus s o(10)$ & $\frac{E_{6}}{\operatorname{Spin}(10)}$ & 33 & Homogeneous CR-manifold \\
\hline (4) & $0^{8} \quad 0^{1}$ & $\varpi_{2}$ & $s o(2) \oplus s o(9)$ & $\frac{E_{6}}{\operatorname{Spin}(9)}$ & 42 & $S^{9}$-bundle over (3) \\
\hline (5) & $0^{8}$ & $\begin{array}{c}\varpi_{1}+\varpi_{3} \\
\left(\varpi_{2}+\varpi_{3}\right)\end{array}$ & $s o(9)$ & $\frac{T \cdot E_{6}}{\operatorname{Spin}(9)}$ & 43 & $\begin{array}{l}P^{2}(\mathbf{O}) \text {-bundle over (2) } \\
\text { (or } S^{1} \text {-bundle over (4)) }\end{array}$ \\
\hline (6) & $0^{1}$ & $\varpi_{1}+\varpi_{2}$ & $s o(8) \oplus s o(2)$ & $\frac{E_{6}}{\operatorname{Spin}(8)}$ & 50 & $S^{8}$-bundle over (4) \\
\hline (7) & $\emptyset$ & $\sum_{i=1}^{3} \varpi_{i}$ & so(8) & $\frac{T \cdot E_{6}}{\operatorname{Spin}(8)}$ & 51 & $S^{1}$-bundle over (6) \\
\hline
\end{tabular}

$\left(F I I=P^{2}(\mathbf{O})=F_{4} / S O(9)\right)$

\begin{tabular}{c|c|c|c|c|c|l}
\hline & $\Pi_{H}$ & $H$ & $\mathfrak{k}_{H}$ & $K / K_{H}$ & $\operatorname{dim} K / K_{H}$ & \\
\hline$(1)$ & $\odot^{8,7}$ & $\{o\}$ & $\operatorname{so}(9)$ & $\{o\}$ & 0 & \\
\hline$(2)$ & $\varnothing$ & $\varpi_{1}$ & $\operatorname{so}(7)$ & $S^{15}=\frac{\operatorname{Spin}(9)}{\operatorname{Sin}(7)}$ & 15 & Symmetric space \\
\hline
\end{tabular}

$\left(G=G_{2} / S O(4)\right.$ as a normal space $)$

\begin{tabular}{c|c|c|c|c|c|c}
\hline & $\Pi_{H}$ & $H$ & $\mathfrak{k}_{H}$ & $K / K_{H}$ & $\operatorname{dim} K / K_{H}$ & \\
\hline$(1)$ & $0^{1}<\equiv \circ^{1}$ & $\{o\}$ & $s o(4)$ & $\{o\}$ & 0 & \\
\hline$(2)$ & $0^{1}$ & $\varpi_{1}\left(\varpi_{2}\right)$ & $s o(2)$ & $\frac{S O(4)}{S O(2)}$ & 5 & Stiefel manifold \\
\hline$(3)$ & $\varnothing$ & $\varpi_{1}+\varpi_{2}$ & 0 & $S O(4)$ & 6 & Symmetric R-space \\
\hline
\end{tabular}

3.4. The classification of local orbit types $\left[\mathfrak{k}_{H}\right]$ of $S$-representations of symmetric R-spaces of classical groups of the rank 2

$(S O(4), r=2)$

\begin{tabular}{c|c|c|c|c|c|c}
\hline & $\Pi_{H}$ & $H$ & $\mathfrak{k}_{H}$ & $K / K_{H}$ & $\operatorname{dim} K / K_{H}$ & \\
\hline$(1)$ & $\circ^{2} \circ^{2}$ & $\{o\}$ & $\operatorname{so}(4)$ & $\{o\}$ & 0 & \\
\hline$(2)$ & $\circ^{2}$ & $\varpi_{1}\left(\varpi_{2}\right)$ & $\mathbf{R} \oplus \operatorname{so}(3)$ & $S^{2}$ & 2 & Symmetric space \\
\hline$(3)$ & $\varnothing$ & $\varpi_{1}+\varpi_{2}$ & $\mathbf{R}^{2}$ & $\frac{S O(4)}{S O(2) \times S O(2)}$ & 4 & Symmetric R-space \\
\hline
\end{tabular}


$(S O(5), r=2)$

\begin{tabular}{c|c|c|c|c|c|c}
\hline & $\Pi_{H}$ & $H$ & $\mathfrak{k}_{H}$ & $K / K_{H}$ & $\operatorname{dim} K / K_{H}$ & \\
\hline$(1)$ & $\circ^{2} \Longrightarrow \circ^{2}$ & $\{o\}$ & $s o(5)$ & $\{o\}$ & 0 & \\
\hline$(2)$ & $\circ^{2}$ & $\varpi_{1}\left(\varpi_{2}\right)$ & $\mathbf{R} \oplus \operatorname{so(3)}$ & $\frac{S O(5)}{S O(2) \times S O(3)}$ & 6 & Symmetric R-space \\
\hline$(3)$ & $\emptyset$ & $\varpi_{1}+\varpi_{2}$ & $\mathbf{R}^{2}$ & $\frac{S O(5)}{S O(2) \times S O(2)}$ & 8 & Flag manifold \\
\hline
\end{tabular}

$(U(3), r=2)$

\begin{tabular}{c|c|c|c|c|c|c}
\hline & $\Pi_{H}$ & $H$ & $\mathfrak{k}_{H}$ & $K / K_{H}$ & $\operatorname{dim} K / K_{H}$ & \\
\hline$(1)$ & $\circ^{2}-\circ^{2}$ & $\{o\}$ & $s u(3)$ & $\{o\}$ & 0 & \\
\hline$(2)$ & $\circ^{2}$ & $\varpi_{1}\left(\varpi_{2}\right)$ & $\mathbf{R} \oplus s u(2)$ & $P^{2}(\mathbf{C})$ & 4 & Symmetric R-space \\
\hline$(3)$ & $\varnothing$ & $\varpi_{1}+\varpi_{2}$ & $\mathbf{R}^{2}$ & $\frac{S U(3)}{S(U(1) \times U(1))}$ & 6 & Flag manifold \\
\hline
\end{tabular}

$(\operatorname{Sp}(2), r=2)$

\begin{tabular}{c|c|c|c|c|c|c}
\hline & $\Pi_{H}$ & $H$ & $\mathfrak{k}_{H}$ & $K / K_{H}$ & $\operatorname{dim} K / K_{H}$ & \\
\hline$(1)$ & $\circ^{2} \Longleftarrow \circ^{2}$ & $\{o\}$ & $s p(2)$ & $\{o\}$ & 0 & \\
\hline$(2)$ & $\circ^{2}$ & $\varpi_{1}\left(\varpi_{2}\right)$ & $\mathbf{R} \oplus \operatorname{sp}(1)$ & $\frac{S O(5)}{S O(2) \times S O(3)}$ & 6 & Symmetric R-space \\
\hline$(3)$ & $\emptyset$ & $\varpi_{1}+\varpi_{2}$ & $\mathbf{R}^{2}$ & $\frac{S O(5)}{S O(2) \times S O(2)}$ & 8 & Flag manifold \\
\hline
\end{tabular}

3.5. The classification of local orbit types $\left[\mathfrak{k}_{H}\right]$ of $S$-representations of symmetric R-spaces of classical groups of the rank 3

$(S O(6), r=3)$

\begin{tabular}{c|c|c|c|c|c|l}
\hline & $\Pi_{H}$ & $H$ & $\mathfrak{k}_{H}$ & $K / K_{H}$ & $\operatorname{dim} K / K_{H}$ & \\
\hline$(1)$ & $\circ^{2}-\circ^{2}-\circ^{2}$ & $\{o\}$ & $s o(6)$ & $\{o\}$ & 0 & \\
\hline$(2)$ & $\circ^{2}-\circ^{2}$ & $\varpi_{1}\left(\varpi_{3}\right)$ & $\mathbf{R} \oplus s u(3)$ & $\frac{S O(6)}{U(3)}$ & 6 & Symmetric R-space \\
\hline$(3)$ & $\circ^{2} \circ^{2}$ & $\varpi_{2}$ & $\mathbf{R} \oplus s o(3)^{2}$ & $\frac{S U(4)}{S(U(2) \times U(2))}$ & 8 & Symmetric R-space \\
\hline$(4)$ & $\circ^{2}$ & $\varpi_{i}+\varpi_{j}(i \neq j)$ & $\mathbf{R}^{2} \oplus s o(3)$ & $\frac{S U(4)}{U(1) \times U(2)}$ & 10 & \\
\hline$(5)$ & $\emptyset$ & $\sum_{i=1}^{3} \varpi_{i}$ & $\mathbf{R}^{3}$ & $\frac{S O(6)}{S O(2)^{3}}$ & 12 & $S^{2}$-bundle over (4) \\
\hline
\end{tabular}


$(S O(7), r=3)$

\begin{tabular}{c|c|c|c|c|c|c}
\hline & $\Pi_{H}$ & $H$ & $\mathfrak{k}_{H}$ & $K / K_{H}$ & $\operatorname{dim} K / K_{H}$ & \\
\hline$(1)$ & $\circ^{2}-\circ^{2} \Longrightarrow \circ^{2}$ & $\{o\}$ & $s o(7)$ & $\{o\}$ & 0 & \\
\hline$(2)$ & $\circ^{2} \Longrightarrow \circ^{2}$ & $\varpi_{1}$ & $\mathbf{R} \oplus \operatorname{so}(5)$ & $\frac{S O(7)}{S O(2) \times S O(5)}$ & 10 & Symmetric R-space \\
\hline$(3)$ & $\circ^{2}-\circ^{2}$ & $\varpi_{3}$ & $\mathbf{R} \oplus s u(3)$ & $\frac{S O(7)}{U(3)}$ & 12 & \\
\hline$(4)$ & $\circ^{2} \circ^{2}$ & $\varpi_{2}$ & $\mathbf{R} \oplus s o(3)^{2}$ & $\frac{S O(7)}{S O(2) \times S O(3)^{2}}$ & 14 & \\
\hline$(5)$ & $\circ^{2}$ & $\varpi_{i}+\varpi_{j}(i \neq j)$ & $\mathbf{R}^{2} \oplus \operatorname{so(3)}$ & $\frac{S O(7)}{S O(2)^{2} \times S O(3)}$ & 16 & $S^{2}$-bundle over (4) \\
\hline$(6)$ & $\varnothing$ & $\sum_{i=1}^{3} \varpi_{i}$ & $\mathbf{R}^{3}$ & $\frac{S O(7)}{S O(2)^{3}}$ & 18 & $S^{2}$-bundle over (5) \\
\hline
\end{tabular}

$(U(4), r=3)$

\begin{tabular}{c|c|c|c|c|c|l}
\hline & $\Pi_{H}$ & $H$ & $\mathfrak{k}_{H}$ & $K / K_{H}$ & $\operatorname{dim} K / K_{H}$ & \\
\hline$(1)$ & $\circ^{2}-\circ^{2}-\circ^{2}$ & $\{o\}$ & $s u(4)$ & $\{o\}$ & 0 & \\
\hline$(2)$ & $\circ^{2}-\circ^{2}$ & $\varpi_{1}\left(\varpi_{3}\right)$ & $\mathbf{R} \oplus s u(3)$ & $P^{3}(\mathbf{C})$ & 6 & Symmetric R-space \\
\hline$(3)$ & $\circ^{2} \circ^{2}$ & $\varpi_{2}$ & $\mathbf{R} \oplus s o(3)^{2}$ & $\frac{S U(4)}{S(U(2) \times U(2))}$ & 8 & Symmetric R-space \\
\hline$(4)$ & $\circ^{2}$ & $\varpi_{i}+\varpi_{j}(i \neq j)$ & $\mathbf{R}^{2} \oplus s o(3)$ & $\frac{S O(6)}{S O(2)^{2} \times S O(3)}$ & 10 & $S^{2}$-bundle over (3) \\
\hline$(5)$ & $\emptyset$ & $\sum_{i=1}^{3} \varpi_{i}$ & $\mathbf{R}^{3}$ & $\frac{S O(6)}{S O(2)^{3}}$ & 12 & $S^{2}$-bundle over (4) \\
\hline
\end{tabular}

$(S p(3), r=3)$

\begin{tabular}{c|c|c|c|c|c|c}
\hline & $\Pi_{H}$ & $H$ & $\mathfrak{k}_{H}$ & $K / K_{H}$ & $\operatorname{dim} K / K_{H}$ & \\
\hline$(1)$ & $\circ^{2}-\circ^{2} \Longleftarrow \circ^{2}$ & $\{o\}$ & $s p(3)$ & $\{o\}$ & 0 & \\
\hline$(2)$ & $\circ^{2} \Longleftarrow \circ^{2}$ & $\varpi_{1}$ & $\mathbf{R} \oplus s p(2)$ & $P^{5}(\mathbf{C})$ & 10 & Symmetric R-space \\
\hline$(3)$ & $\circ^{2}-\circ^{2}$ & $\varpi_{3}$ & $\mathbf{R} \oplus s u(3)$ & $\frac{S p(3)}{U(3)}$ & 12 & Symmetric R-space \\
\hline$(4)$ & $\circ^{2} \circ^{2}$ & $\varpi_{2}$ & $\mathbf{R} \oplus s o(3)^{2}$ & $\frac{S p(3)}{S^{1} \times S p(1)^{2}}$ & 14 & \\
\hline$(5)$ & $\circ^{2}$ & $\varpi_{i}+\varpi_{j}(i \neq j)$ & $\mathbf{R}^{2} \oplus s o(3)$ & $\frac{S p(3)}{S O(2)^{2} \times S O(3)}$ & 16 & $S^{2}$-bundle over (4) \\
\hline$(6)$ & $\emptyset$ & $\sum_{i=1}^{3} \varpi_{i}$ & $\mathbf{R}^{3}$ & $\frac{S p(3)}{S O(2)^{3}}$ & 18 & $S^{2}$-bundle over (5) \\
\hline
\end{tabular}


3.6. The classification of local orbit types $\left[\mathfrak{k}_{H}\right]$ of $S$-representations of the real quadrics $S O(p+1) \times S O(q+1) / S(O(p) \times O(q)), p \leq q$

\begin{tabular}{c|c|c|c|c|c|c}
\hline & $\Pi_{H}$ & $H$ & $\mathfrak{k}_{H}$ & $K / K_{H}$ & $\operatorname{dim} K / K_{H}$ & \\
\hline$(1)$ & ${ }^{p-1}{ }^{q-1}$ & $\{o\}$ & $\operatorname{so}(p) \oplus \operatorname{so}(q)$ & $\{o\}$ & 0 & \\
\hline$(2)$ & ${ }^{q-1}$ & $\varpi_{1}$ & $\operatorname{so}(p-1) \oplus \operatorname{so}(q)$ & $S^{p-1}$ & $p-1$ & Symmetric space \\
\hline$(3)$ & ${ }^{p-1}$ & $\varpi_{2}$ & $\operatorname{so}(q-1) \oplus \operatorname{so}(p)$ & $S^{q-1}$ & $q-1$ & Symmetric space \\
\hline$(4)$ & $\emptyset$ & $\varpi_{1}+\varpi_{2}$ & $s o(p-1) \oplus \operatorname{so}(q-1)$ & $S^{p-1} \times S^{q-1}$ & $p+q-2$ & Symmetric space \\
\hline
\end{tabular}

\section{References}

[HeiOlm] E. HeIntzE and C. OLmos, Normal holonomy groups and s-representations, Indiana Univ. Math. J. 41 (1992), 869-874.

[Hel] S. Helgason, Differential Geometry, Lie Groups, and Symmetric Spaces, Academic Press, (1978).

[TaKo] M. TAKeUChI and S. KobaYAShi, Minimal imbeddings of R-spaces, J. Diff. Geom. 2 (1968), $203-215$.

[Tama1] H. TAMARU, The orbit types of s-representations, unpublished (1997).

[Tama2] H. TAMARU, The local orbit types of symmetric spaces under the actions of the isotropy subgroups, Diff. Geom. Appl. 11 (1999), 29-38.

Present Address:

Department of MATHEMATiCs, FACUlty of SCIENCE AND ENGINEERING,

SAGA UNIVERSITY, HONJOH, SAGA, 840-8502 JAPAN.

e-mail:kondok@ms.saga-u.ac.jp 